\title{
Adjustment Reaction with Brief Depressive Reaction
}

National Cancer Institute

\section{Source}

National Cancer Institute. Adjustment Reaction with Brief Depressive Reaction. NCI

Thesaurus. Code C34358.

Temporary maladaptive depressive signs and symptoms associated with a life event. 